\title{
Spinal Accessory Nerve Palsy following Cervical Cooled RADIOFREQUENCY ABLATION: A CASE REPORT AND REVIEW OF THE LITERATURE
}

Neelay S. Thakkar, MD¹, Matthew J. Gray, MD², Shilpa S. Ramesh, MD², John H. Vetter, MD', and Tristan E. Weaver, MD²

Chronic neck pain is a common cause of functional impairment in the general population. A significant percentage has a component of cervical facet arthropathy for which cervical radiofrequency ablation (RFA) has been successful in treating. We present a case of spinal accessory nerve (SAN) palsy after water-cooled cervical RFA.

A 37-year-old female with history of fibromyalgia and occipital neuralgia presented with cervicalgia. Magnetic resonance imaging (MRI) revealed degenerative changes and central canal stenosis at C5-6. After positive result to diagnostic cervical medial branch blocks (MBB), she underwent staged bilateral C2-3-4-5 medial branch watercooled RFA. On subsequent follow up, she noted new left shoulder pain. On exam, difficulty with left arm abduction and scapular winging was noted. Electrodiagnostics (EDX) revealed mild denervation and mild decreased motor units on needle EMG study. Nerve stimulation study of the SAN to the upper trapezius revealed latency prolongation and amplitude reduction, consistent with an acute spinal accessory neuropathy. Repeat EDX study, 7 months later, no longer showed denervation in upper trapezius, normal latency, and improved (although still decreased) amplitude.

SAN palsy after multilevel cervical RFA has not been reported in the literature to our knowledge. Cervical RFA is generally considered safe with most complications being transient and minor with no lasting adverse effects. Spinal accessory nerve palsy is one cause of scapular winging. This case highlights the importance of physical exam and knowledge of anatomical structures in promptly diagnosing SAN palsy.

Key words: Spinal accessory nerve palsy, medial scapular winging, lateral scapular winging, cervical radiofrequency ablation, cervical facet arthropathy, cervical medial branch blocks, cervicalgia, water-cooled radiofrequency ablation
Radiofrequency ablation (RFA) is a minimally invasive procedure that uses thermal energy to ablate target sensory nerve fibers (1). Multiple studies have proven that RFA provides longer-term pain relief over more conventional intra-articular facet injections. RFA is now a commonly performed procedure for patients

From : 'Department of Physical Medicine and Rehabilitation, The Ohio State University Wexner Medical Center; ${ }^{2}$ Department of Anesthesiology, The Ohio State University Wexner Medical Center

Author for correspondence: Tristan Weaver, MD

Address: Department of Anesthesiology, The Ohio State University Wexner Medical Center, 543 Taylor Ave., Columbus, OH 43203

E-mail: tristan.weaver@osumc.edu presenting with facet-related axial back or nexk pain. Standard RFA causes focal nerve denervation when the active tip is placed in close proximity to the target neural structure, but many interventionalists have recently incorporated a more novel technique of water-cooled RFA. This differs from standard RFA in that the cooled-RFA probe allows for a larger lesion to be created, thereby improving the chances of target nerve denervation and producing a more effective relief of pain (2). This case demonstrates the first documented complication of a spinal accessory nerve (SAN) palsy resulting in lateral scapular winging following cervical water-cooled RFA for facet arthropathy. 


\section{Case Presentation}

A 37-year-old female with past medical history of celiac disease, depression, fibromyalgia, nephrolithiasis, occipital neuralgia, postural orthostatic tachycardia syndrome, and small fiber neuropathy, presented in October 2015 with a chronic history of cervicalgia. The patient reported axial neck pain, along with radicular pain into the bilateral upper limbs, predominantly on the right side. She described the quality of her pain to be sharp and shooting in nature. The patient also reported moderately severe paresthesia on the lateral aspect of her hands and subjective weakness in handgrip strength bilaterally. Alleviating factors included rest and cryotherapy; neck extension and rotation exacerbated her pain. Her symptoms had worsened in the last year after pregnancy and subsequent childbirth. She reported seeing a neurologist in the past who noted this to be likely myofascial in origin. She also had a history of improvement with occipital nerve blocks 6 years ago. She had no benefit from various medications including: gabapentin, tapentadol, cyclobenzaprine, acetaminophen, and ibuprofen. The patient did not have a history of cervical surgery.

On physical exam, tenderness to palpation was noted over bilateral cervical paraspinals and the occipital ridge. There was also positive facet-loading bilaterally. There was no sensory loss or motor weakness in upper extremities. Magnetic resonance imaging (MRI) revealed degenerative changes most pronounced at C5-C6 with moderate bilateral neuroforaminal narrowing, as well as mild central canal stenosis at C5-C6 and $\mathrm{C} 6-\mathrm{C} 7$ levels. Mild stable spondylolisthesis at C5-C6 was noted on plain x-ray flexion-extension imaging. An electrodiagnostic (EDX) study revealed normal EDX findings of the bilateral upper limbs without evidence of cervical radiculopathy, cervical brachial plexopathy, carpal tunnel syndrome, or an ulnar neuropathy across the elbow.

Bilateral occipital nerve injections were repeated in December 2015. The patient's symptoms did not resolve and it was determined that there was likely a myofascial component with facet arthropathy contributing to the pain. She therefore underwent right C2-3-4-5 diagnostic medial branch blocks (MBB) performed January 2016 and returned 2 weeks later for the same procedure on the left. Given improvement greater than $50 \%$ with the MBB, she underwent water-cooled RFA of the C2-3-4-5 medial branches. The right side was performed in April 2016 and the left was performed 2 weeks later (Fig. 1).

At a subsequent follow-up visit, she reported new left shoulder pain that had progressively worsened over the past couple months. She described the pain as a constant aching and sharp/stabbing. The patient was started on a trial of pregabalin. An MRI of the left shoulder was obtained, which revealed mild tendinosis of the infraspinatus and supraspinatus tendons with partial articular sided tear of supraspinatus and she was referred to orthopedic surgery for further evaluation. The patient then underwent $\mathrm{C} 7 /$ T1 interlaminar epidural steroid injection for cervical radiculitis and degenerative changes at C5-6; however, this failed to significantly improve symptoms. There was also evidence of atrophy limited only to the left upper trapezius muscle and scapular winging on physical exam (Fig. 2). An electromyography (EMG) test performed in July 2016 revealed acute changes with both positive sharp waves and fibrillations, and decreased motor units in the left trapezius (Table 1). Nerve stimulation study revealed a prolonged latency of the SAN to the upper trapezius as well as a decreased amplitude (comparison was made to opposite unaffected side). This was consistent with acute SAN neuropathy.

Throughout the patient's course, she was prescribed physical therapy and recommended to continue a home exercise plan for scapular stabilization techniques. The patient's neurologist noted borderline paraneoplastic antibody and ordered imaging to evaluate for possible nerve compression by soft tissue mass. MRI of the brachial plexus and neck revealed normal appearance of the brachial plexus with no overt atrophy of the left trapezius compared to the right. An EDX study performed in December 2016 revealed significant improvement with no denervation seen. Also, the nerve latency of the SAN to the upper trapezius was now normal and the amplitude had improved.

\section{Discussion}

Rarely has SAN palsy been seen to occur following RFA as presented in the above case (3). The SAN is the most susceptible cranial nerve to injury due to its superficial course in the posterior triangle, particularly following surgical procedures involving this area $(4,5)$. 
The SAN arises from the cranial nerve fibers and upper 5 or 6 cervical nerve roots (4). Diagnosis of SAN palsy is typically based on history and physical examination findings followed by an EDX study confirmation. SAN palsy leads to weakness of the trapezius muscle, which usually causes winging of the scapula along with mild to moderate impairment (5). An MRI may reveal muscle abnormalities in greater than $50 \%$ of cases. Awareness of this condition is crucial, as proper diagnosis will help in proper management.

Early diagnosis requires a keen eye on the potential physical exam findings, potentially indicative of SAN palsy, such as winging of the scapula and/or atrophy of the upper trapezius muscle in particular. Scapular winging results from an imbalance of muscles responsible for stabilizing the scapula and typically occurs from a lesion of either the long thoracic nerve or SAN (5). Each nerve provides innervation to different muscles resulting in a unique presentation seen with injury; thus, a thorough understanding of the differences seen with each lesion can aid a clinician in making the proper diagnosis. An injury to the SAN nerve results in weakness of the trapezius muscle and is particularly at risk to injury from blunt trauma or iatrogenic causes, such as lymph node biopsy or mass excision. The superior and inferior fibers of the trapezius are responsible for elevation and lateral rotation of the scapula. Therefore, on exam one will find the superior medial scapula is downward and lateral, resulting in lateral winging of the scapula following SAN palsy.

Injury to the long thoracic nerve results in weakness of the serratus anterior, which acts primarily to stabilize and protract the scapula and is most commonly injured following blunt trauma or a stretch injury. Potential iatrogenic causes also include: anterior cervical decompression, mastectomy, axillary dissection, positioning during surgery, and thoracostomy tube placement. Due to the significant contribution from the $\mathrm{C} 7$ nerve root, injury has also been reported to occur following a $\mathrm{C} 7$ radiculopathy (6). Weakness of the serratus anterior muscle results in medial winging of the scapula, which differentiates it from SAN palsy. The medial winging is accentuated by forward flexion compared to abduction with lateral winging (5). This illustrates the importance of performing a proper physical exam when diagnosing scapular winging.

Many cases of scapular winging will improve over time. Initial management is therefore non-surgical
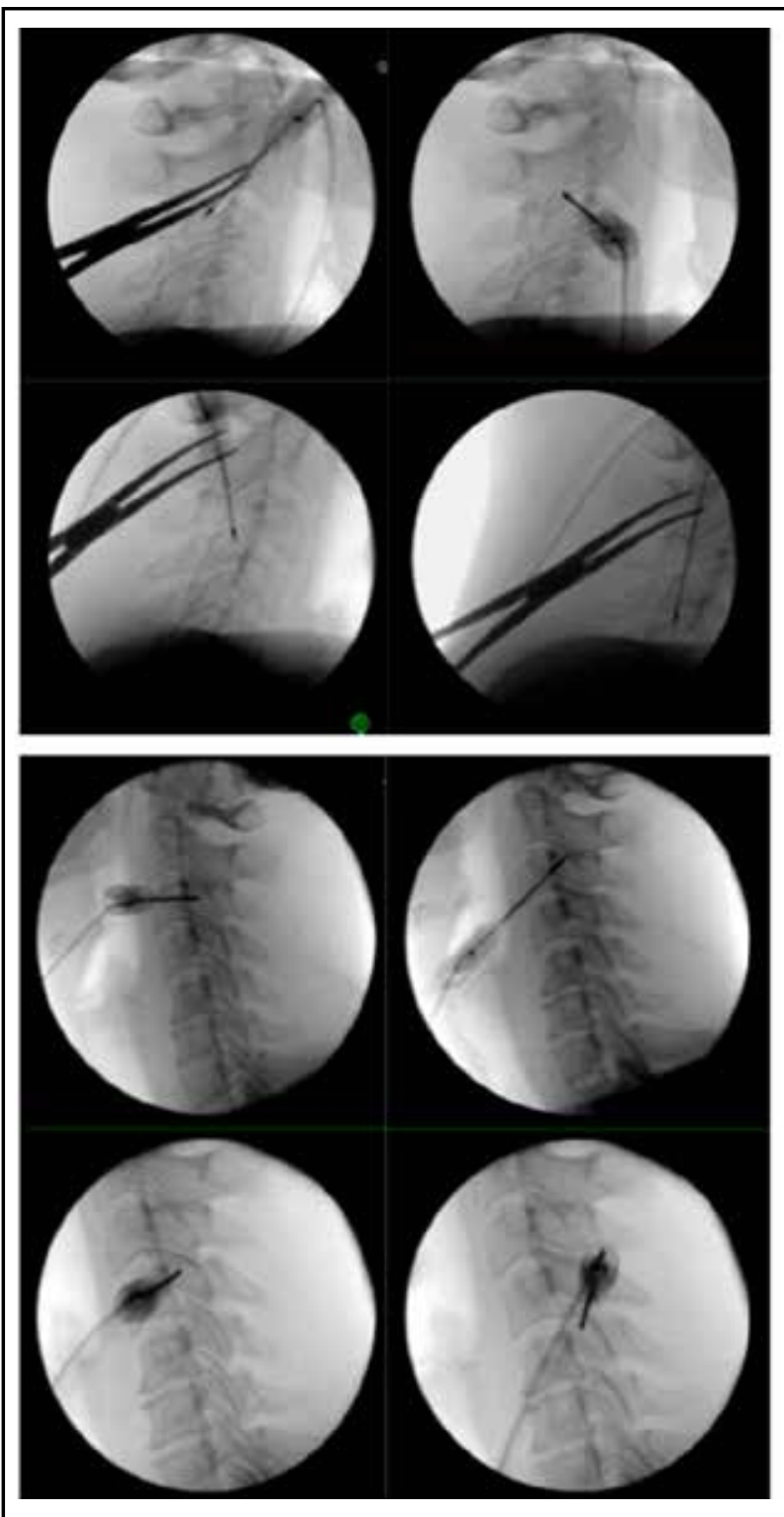

Fig. 1. X-ray imaging of water-cooled RFA.

Top: Right C2-3-4-5. Bottom: Left C2-3-4-5. The patient was placed in the lateral decubitus position. The centroid of the lateral mass of the $\mathrm{C} 2,3,4,5$ vertebrae were targeted utilizing 17 gauge $5 \mathrm{~cm}$ water-cooled RFA needle introducers. AP and lateral views were utilized to confirm placement. Ablation for 2.5 minutes to $60^{\circ} \mathrm{C}$ occured at each level.

with activity modification, physical/occupational therapy, and analgesics. The patient should be cautioned to avoid carrying heavy objects on the affected side. Additional modalities, such as neuromuscular electrical stimulation and stretching/strengthening programs, are also beneficial (4). However, in the case that penetrating trauma is suspected, surgical 


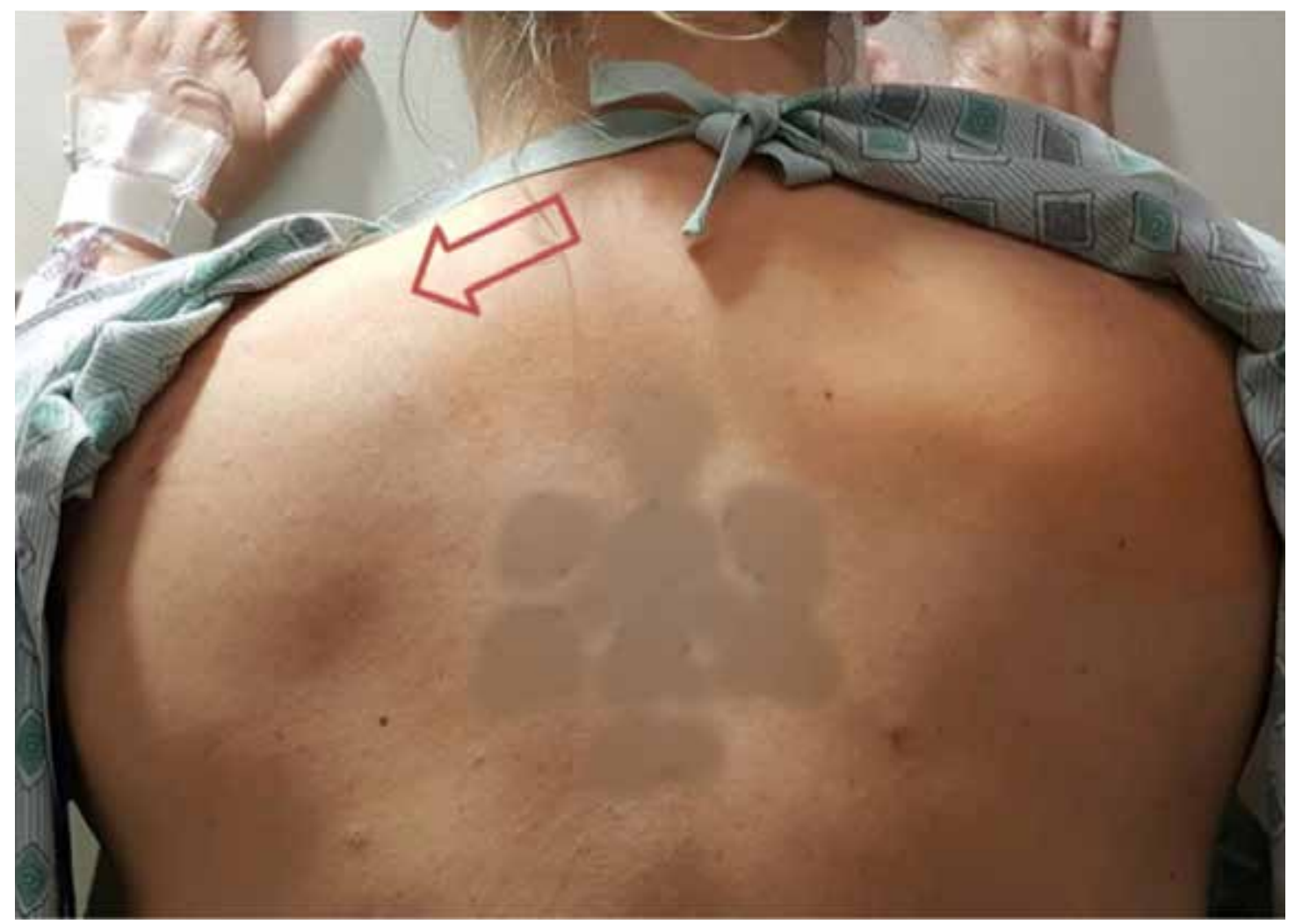

Fig. 2. Evidence of lateral winging with slight atrophy of the left trapezius compared to the right. Consent obtained from patient for use of picture and identifying features have been obscured.

exploration with neurolysis or nerve grafting may be indicated (3).

Due to its effectiveness, cervical RFA has been used to treat neck and back pain since the 1970s. Studies have shown that it results in $63 \%$ of patients being pain free at 6 months and $38 \%$ pain free at 1 year (3). The effectiveness is entirely dependent on adhering to current published guidelines when performing the procedure, as well as after positive diagnostic blocks have been done (3). A positive response to MBB is typically defined as greater than $50 \%$ pain reduction, which lasts the average duration of the local anesthetic that was used (3). Most of the current literature related to RFA treatment is limited to 1 year follow-up with the longest known follow-up interval reported at 3 years. The nerves remain intact anatomically and can recover over time, thereby requiring repeat treatments. A major influence on the duration of effectiveness is determined by the full-thickness length of the target nerve coagulated during the RFA treatment (3). Cooled RFA differs from standard RFA in that water is run through the probe tip in order to maintain a cooler temperature. The temperature is typically maintained between $50-60^{\circ} \mathrm{C}$, whereas in standard RFA the probe is heated to $80^{\circ} \mathrm{C}$. Internal cooling also results in less charring of adjacent tissue, which allows for more effective ionic heating of the tissue further from the probe. One study demonstrated that the average lesion size of standard RFA was a length of $10 \mathrm{~mm}$ and a width of $4 \mathrm{~mm}$ with an elliptical shape. The average lesion size of cooled RFA is larger at a length of $10 \mathrm{~mm}$ and a width of 10 $\mathrm{mm}$ with a circular shape (Fig. 3) (7).

Potential complications of cervical RFA include: infection, bleeding, increased pain, vasovagal reaction, bruising, ataxia, and spinal nerve lesioning $(3,8)$. However, there are reports in the literature of side effects and complications from cervical RFA 
Table 1: Initial NCS/EMG: Prolonged latency and decreased amplitude of the left upper trapezius muscle. Positive sharp waves and fibrillation potentials isolated in the left upper trapezius muscle. These findings with no other abnormalities are suggestive of left spinal accessory nerve palsy. Repeat study with estimated $60 \%$ improvement in amplitude of left upper trapezius muscle.

\begin{tabular}{|c|c|c|c|}
\hline Nerve Conduction Study & $\begin{array}{c}\text { Right } \\
\text { (latency/amplitude) }\end{array}$ & $\begin{array}{c}\text { Left } \\
\text { (latency/amplitude) }\end{array}$ & Normal \\
\hline Radial Sensory to Thumb@14 cm & & $3.0 \mathrm{~ms} / 10 \mu \mathrm{V}$ & $<3.6 \mathrm{msec}$ \\
\hline Median Sensory to Index Finger @ 14 cm & & $3.1 \mathrm{~ms} / 60 \mu \mathrm{V}$ & $<3.6 \mathrm{msec}$ \\
\hline Median Sensory to Middle Finger @ $14 \mathrm{~cm}$ & & $3.0 \mathrm{~ms} / 10 \mu \mathrm{V}$ & $<3.6 \mathrm{msec}$ \\
\hline Ulnar Sensory to Small Finger @ $14 \mathrm{~cm}$ & & $3.0 \mathrm{~ms} / 20 \mu \mathrm{V}$ & $<3.6 \mathrm{msec}$ \\
\hline Median Palmer@8cm & & $1.9 \mathrm{~ms} / 60 \mu \mathrm{V}$ & $<2.4 \mathrm{msec}$ \\
\hline UlnarPalmer@8 cm & & $2.1 \mathrm{~ms} / 20 \mu \mathrm{V}$ & $<2.4 \mathrm{msec}$ \\
\hline Median Motor@ Wrist & & $2.9 \mathrm{~ms} / 6.1 \mu \mathrm{V}$ & $<4.3 \mathrm{msec}$ \\
\hline Median NCV: below elbow to wrist & & $68 \mathrm{~m} / \mathrm{s}$ & $>45 \mathrm{~m} / \mathrm{s}$ \\
\hline Ulnar Motor@Wrist & & $2.7 \mathrm{~ms} / 9.0 \mu \mathrm{V}$ & $<3.8 \mathrm{msec}$ \\
\hline Ulnar NCV: below elbow to wrist & & $8.5 \mathrm{~ms} / 68 \mathrm{~m} / \mathrm{s}$ & $>45 \mathrm{~m} / \mathrm{s}$ \\
\hline Ulnar NCV: above elbow to below elbow & & $8.3 \mathrm{~ms} / 69 \mathrm{~m} / \mathrm{s}$ & $>45 \mathrm{~m} / \mathrm{s}$ \\
\hline Spinal Accessory Nerve to mid-upper Trapezius @ $10 \mathrm{~cm}$ & & & $<3.0 \mathrm{msec}$ \\
\hline Initial & $2.9 \mathrm{~ms} / 6.9 \mathrm{mV}$ & $3.4 \mathrm{~ms} / 970 \mu \mathrm{V}$ & \\
\hline Follow-up 7 months later & $2.6 \mathrm{~ms} / 6.1 \mathrm{mV}$ & $2.7 \mathrm{~ms} / 1.6 \mathrm{mV}$ & \\
\hline * Temperature $34.8^{\circ} \mathrm{C}$ & & & \\
\hline
\end{tabular}

\begin{tabular}{|c|c|c|c|c|c|}
\hline Muscle & Innervation & $\begin{array}{c}\text { Insertional } \\
\text { Activity }\end{array}$ & Fibrillations & Fasciculations & $\begin{array}{c}\text { Motor Unit Action } \\
\text { Potentials }\end{array}$ \\
\hline Left Upper Trapezius & C 2,3,4 Spinal Accessory N. & $\begin{array}{c}1-2+\text { positive } \\
\text { waves }\end{array}$ & $1+$ & $\begin{array}{c}\text { Mild decreased number } \\
\text { with normal amplitude } \\
\text { and duration. }\end{array}$ \\
\hline $\begin{array}{c}\text { Left Supraspinatus/ } \\
\text { Infraspinatus }\end{array}$ & C 5,6 Suprascapular N. & Normal & 0 & 0 & \\
\hline Left Deltoid & C 5,6 Axillary N. & Normal & 0 & 0 & \\
\hline Left Biceps & C 5,6 Musculocutaneous N. & Normal & 0 & 0 & \\
\hline Left Triceps & C 6,7,8 Radial N. & Normal & 0 & 0 & \\
\hline Left Pronator Teres & C 6,7 Median N. & Normal & 0 & 0 & 0 \\
\hline Left Ext Dig Comm. & C 7,8 Radial N. & Normal & 0 & 0 & \\
\hline Left 1st Dorsal Inter. & C8, T1 Ulnar N. & Normal & 0 & 0 & \\
\hline Left Abd. Dig. Minimi & C8, T1 Ulnar N. & Normal & 0 & 0 & 0 \\
\hline Left Abd. Poll. Brevis & C8, T1 Median N. & Normal & 0 & 0 & \\
\hline $\begin{array}{c}\text { Left Cervical Paraspinals } \\
\text { (C4-T1) }\end{array}$ & Posterior Rami & Normal & 0 & 0 & 0 \\
\hline
\end{tabular}

performed in ways that did not strictly follow current guidelines. This includes multiple cases of neuritis, shown to occur after RFA of third occipital nerve, possibly due to its cutaneous component $(3,9)$. Another is a case of Horner's syndrome, which was first described in 2014 following a C5-6-7 RFA that spontaneously resolved after a period of 36 hours with no lasting effects (10). Dropped head syndrome or postoperative kyphosis due to extensor muscle denervation, has been reported twice in patients treated by cervical RFN at multiple levels. In both cases, the patients required treatment by surgi- 


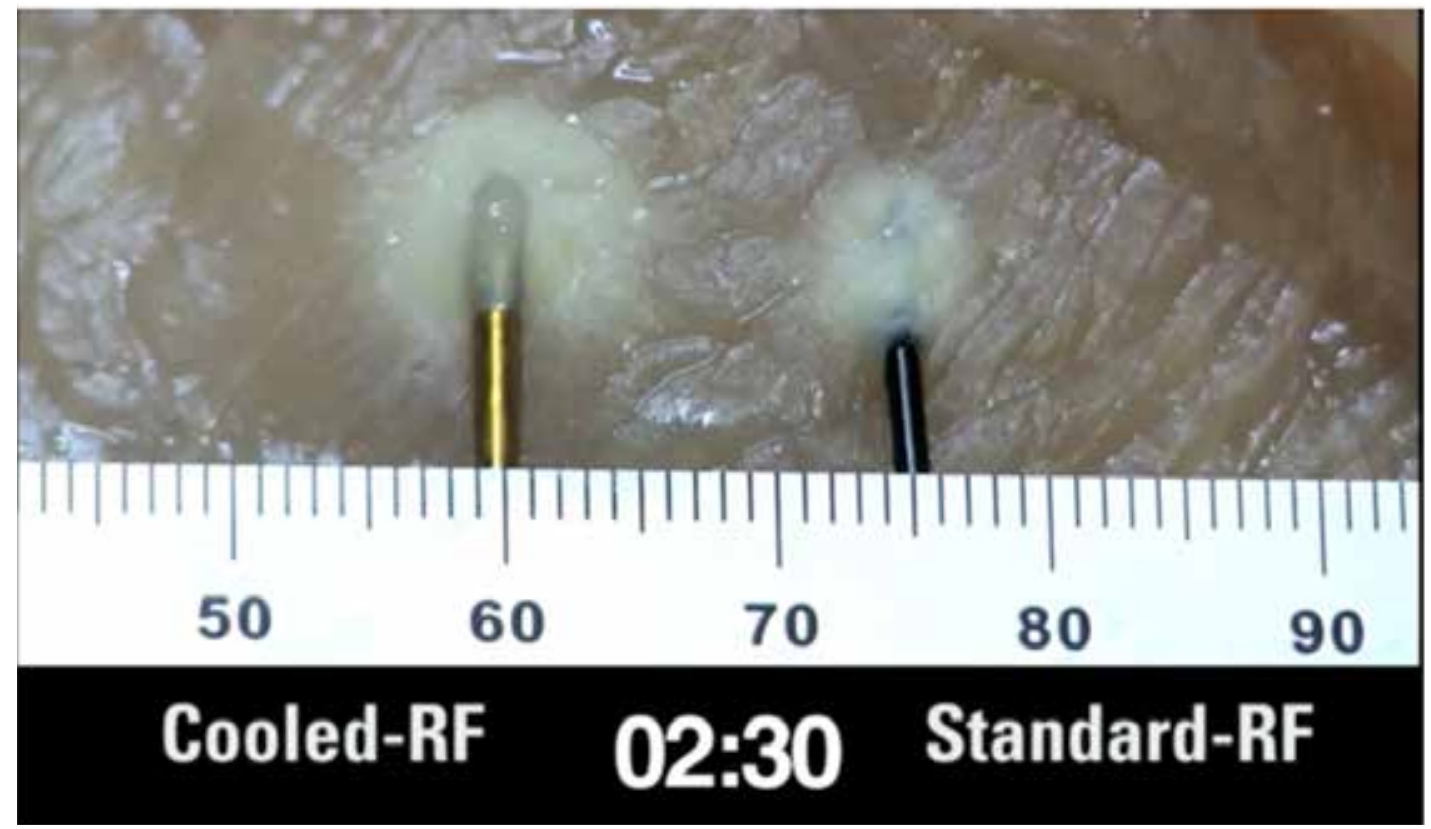

Fig. 3. Comparison of cooled and standard RFA lesion size.

cal fusion. It is suspected to have occurred due to denervation of cervical extensor muscles from too many levels being treated $(11,12)$. There have also been reported cases of neural and vascular injury following cervical RFA.

One case of Brown-Séquard syndrome developed following RFA of a C3-4 facet joint. Intraoperative films revealed that electrodes were placed medial to the target joint, thereby passing between the laminae and entering the spinal cord. The second case involved a spinal cord infarction following RFA of a C2-3 facet joint. Films later revealed the electrode to be lying in the C3-4 intervertebral foramen, instead of the C2-3 foramen. In both instances, the procedure had been performed under general anesthesia and therefore the patient was not able to report any unusual sensations (13).

In conclusion, cervical RFA is a commonly performed procedure for facet mediated neck pain with most risks and complications being minor and transient. While other rare and serious complications have been reported in the literature, to our knowledge this is the first reported case of SAN palsy following cervical RFA. A thorough understanding of the anatomy and physical examination is essential to make a prompt diagnosis of SAN palsy. Though it is rare, clinicians and patients should be aware of this potential complication when considering cervical RFA. 


\section{REFERENCES}

1. Ho KY, Hadi MA, Pasutharnchat $\mathrm{K}$, Tan $\mathrm{KH}$. Cooled radiofrequency denervation for treatment of sacroiliac joint pain: Twoyear results from 20 cases. J Pain Res 2013; 6:505-511.

2. McCormick ZL, Walker J, Marshall B, McCarthy R, Walega DR. A novel modality for facet joint denervation: Cooled radiofrequency ablation for lumbar facet syndrome. A case series. Phys Med Rehabil Int 2014; 1:5.

3. Engel A, Rappard G, King W, Kennedy DJ, Society SDotISI. The effectiveness and risks of fluoroscopically-guided cervical medial branch thermal radiofrequency neurotomy: A systematic review with comprehensive analysis of the published data. Pain Med 2016; 17:658-669.

4. Ozdemir O, Kurne A, Temuçin C, Varli K. Spontaneous unilateral accessory nerve palsy: A case report and review of the literature. Clin Rheumatol 2007; 26:1581-1583

5. Gooding BW, Geoghegan JM, Wallace WA, Manning PA. Scapular winging. Shoulder Elbow 2014; 6:4-11.

6. Meininger AK, Figuerres BF, Goldberg BA. Scapular winging: An update. J Am Acad Orthop Surg 2011; 19:453-462.

7. Cohen SP, Hurley RW, Buckenmaier CC 3rd, Kurihara C, Morlando B, Dragovich A. Randomized placebo-controlled study evaluating lateral branch radiofrequency denervation for sacroiliac joint pain. Anesthesiology 2008; 109:279-288.

8. Govind J, King W, Bailey B, Bogduk N. Radiofrequency neurotomy for the treatment of third occipital headache. J Neurol Neurosurg Psychiatry 2003; 74:88-93.

9. Gazelka HM, Knievel S, Mauck WD, Moeschler SM, Pingree MJ, Rho RH, Lamer TJ. Incidence of neuropathic pain after radiofrequency denervation of the third occipital nerve. J Pain Res 2014; 7:195-198.

10. Kafafy A. Horner syndrome after cervical median branch radiofrequency neurotomy. ERSA Academy September 3, 2015.

11. Ahmed MM, Lake WB, Resnick DK. Progressive severe kyphosis as a complication of multilevel cervical percutaneous facet neurotomy: A case report. Spine ] 2012; 12:e5-e8.

12. Stoker GE, Buchowski JM, Kelly MP. Dropped head syndrome after multilevel cervical radiofrequency ablation: A case report. J Spinal Disord Tech 2013; 26:444-448.

13. Bogduk N, Dreyfuss $P$, Baker R, Yin W, Landers M, Hammer MA, Aprill C. Complications of spinal diagnostic and treatment procedures. Pain Medicine 2008; 9:S11-S34. 
